\title{
X-ray study of anisotropically shaped metal halide perovskite nanoparticles in tubular pores
}

Janina Melanie Roemer, Stepan Demchyshyn, Anton Böhm, Olof Gutowski, Kilian Frank, Niyazi Serdar Sariciftci, Martin Kaltenbrunner, and Bert Nickel

Citation: Appl. Phys. Lett. 113, 251901 (2018); doi: 10.1063/1.5054271

View online: https://doi.org/10.1063/1.5054271

View Table of Contents: http://aip.scitation.org/toc/apl/113/25

Published by the American Institute of Physics

\section{Articles you may be interested in}

Improved performance of InP-based $2.1 \mu \mathrm{m} \operatorname{InGaAsSb}$ quantum well lasers using $\mathrm{Sb}$ as a surfactant Applied Physics Letters 113, 251101 (2018); 10.1063/1.5060653

Short infrared wavelength quantum cascade detectors based on $\mathrm{m}$-plane $\mathrm{ZnO} / \mathrm{ZnMgO}$ quantum wells Applied Physics Letters 113, 251104 (2018); 10.1063/1.5058120

High-efficiency shallow-etched grating on GaAs membranes for quantum photonic applications Applied Physics Letters 113, 251103 (2018); 10.1063/1.5055622

Measurement of ultrafast dynamics of photoexcited carriers in $\beta-\mathrm{Ga}_{2} \mathrm{O}_{3}$ by two-color optical pump-probe spectroscopy

Applied Physics Letters 113, 252102 (2018); 10.1063/1.5058164

Enhanced response of bulk heterojunction polymer photodetectors upon incorporating $\mathrm{CsPbBr} 3$ quantum dots Applied Physics Letters 113, 253301 (2018); 10.1063/1.5050253

Exciton transport in strained monolayer WSe2

Applied Physics Letters 113, 252101 (2018); 10.1063/1.5063263

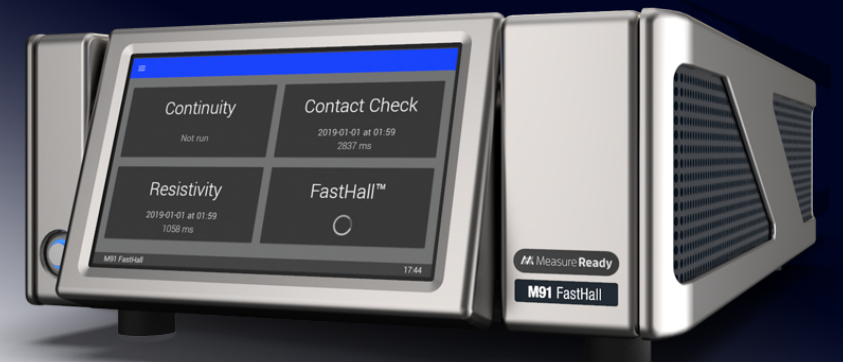

\section{m Measure Ready} M91 FastHall ${ }^{\mathrm{TM}}$ Controller

A revolutionary new instrument for complete Hall analysis 


\title{
X-ray study of anisotropically shaped metal halide perovskite nanoparticles in tubular pores
}

\author{
Janina Melanie Roemer, ${ }^{1}$ Stepan Demchyshyn, ${ }^{2,3}$ Anton Böhm, ${ }^{1}$ Olof Gutowski, ${ }^{4}$ \\ Kilian Frank, ${ }^{1}$ Niyazi Serdar Sariciftci, ${ }^{2}$ Martin Kaltenbrunner, ${ }^{3}$ and Bert Nickel ${ }^{1,5}$, a) \\ ${ }^{1}$ Faculty of Physics and CeNS, Ludwig-Maximilians-University München, Geschwister-Scholl-Platz 1, \\ 80539 Munich, Germany \\ ${ }^{2}$ Linz Institute for Organic Solar Cells (LIOS), Physical Chemistry, Johannes Kepler University Linz, \\ Altenbergerstr. 69, 4040 Linz, Austria \\ ${ }^{3}$ Soft Electronics Laboratory, Linz Institute of Technology LIT, Johannes Kepler University Linz, \\ Altenbergerstr. 69, 4040 Linz, Austria \\ ${ }^{4}$ DESY Photon Science, Notkestr. 85, 22603 Hamburg, Germany \\ ${ }^{5}$ Nanosystems Initiative Munich, Schellingstr. 4, 80799 München, Germany
}

(Received 30 August 2018; accepted 2 December 2018; published online 19 December 2018)

\begin{abstract}
Recently, we have reported that metal halide perovskite nanoparticles formed in nanoporous alumina and silicon thin films exhibit blue shifted photoluminescence due to spatial confinement, thus allowing for color tuning of the emission by varying the pore size. While perovskite nanoparticles grown in nanoporous alumina films have been integrated into LEDs, similar approaches have failed with silicon. Here, we report the results of investigating the structure of the alumina pore system and the perovskite crystallites forming within. We use two x-ray diffraction techniques, namely, small-angle x-ray scattering (SAXS) and high-energy microbeam wide-angle $\mathrm{x}$-ray scattering (WAXS). SAXS reveals that the alumina pore system diffracts like regularly arranged tubes with the average diameter and nearest neighbor distance of $12 \mathrm{~nm}$ and $20 \mathrm{~nm}$, respectively. High-energy microbeam WAXS shows that perovskite nanoparticles within the nanoporous alumina have a distinctly anisotropic shape with the average particle length along and perpendicular to the pore axis of $26 \mathrm{~nm}$ and $13 \mathrm{~nm}$, respectively. In contrast, no shape anisotropy has been detected for nanoparticles inside the silicon pores in a previous study. This suggests that utilizing nanoporous alumina has a twofold advantage. First, the tubular alumina pores, spanning the entire insulating film, offer percolated paths for the perovskite to fill. Second, the elongation of the nanoparticles in the tubular alumina pores can be expected to aid device performance as the length of the nanoparticles approaches the active layer thickness (ca. $40 \mathrm{~nm}$ ) of LEDs, while the small diameter of the crystallites accounts for the observed blue shifted emission. Published by AIP Publishing.

https://doi.org/10.1063/1.5054271
\end{abstract}

Quantum confinement of metal halide perovskites can be achieved via size control, allowing for tuning of optical properties. ${ }^{1-5}$ The synthesis of size controlled perovskite nanoparticles (NPs) can be divided into colloidal and template assisted methods. ${ }^{6}$ Colloidal synthesis yields ligand functionalized nanoparticles which require further steps in order to be integrated into optoelectronic devices. ${ }^{7-9}$ In template assisted synthesis, NPs nucleate directly within porous particles $^{10,11}$ or thin films. ${ }^{12-14}$ In particular, thin films can be directly integrated into optoelectronic devices, owing to mild synthesis conditions and high compatibility with prepatterned contact structures. The influence of the porous template on the NP size and nucleation density of perovskites was explored by several groups in parallel. ${ }^{10,11,14}$ In particular, nanoporous silicon (npSi) allows for pore sizes down to few nanometers. In a previously published study, ${ }^{14}$ we observed pronounced photoluminescence (PL) blue shifts of up to $151 \mathrm{~nm}$ for perovskite NP in npSi compared to perovskite bulk films. Using $\mathrm{x}$-ray analysis, we could quantify the relation between pore size, NP size, and PL shift. Perovskites in nanoporous anodic alumina (npAl) thin films

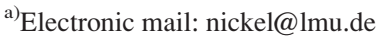

showed PL shifts of up to $62 \mathrm{~nm} .{ }^{14}$ While perovskite NP within npSi proved to be challenging to address electrically, npAl was readily used as a scaffold for perovskite NP active layers in light-emitting diodes (LEDs). ${ }^{14}$ Here, we explore the nanoscale structural differences which enable the integration of npAl films into devices. Since the empty npAl scaffolds are electrically insulating, we hypothesize that, in an operating device, electron and hole transport across the scaffold occurs exclusively via the perovskite NPs, which also promote recombination. Therefore, insights into the geometry of the perovskite NP and the pores are essential for understanding and improving device performance. We aim to provide this structural information by the use of two complementary x-ray scattering techniques. This allows us to simultaneously probe millions of pores or NP, respectively, providing comprehensive insights into the universal properties of the layers. ${ }^{15}$ Small-angle $\mathrm{x}$-ray scattering (SAXS) is used to investigate the morphology of the nanoporous layers. Information about the perovskite NP within the pores is obtained via high-energy wide-angle $\mathrm{x}$-ray scattering (WAXS).

We prepare the samples as described in Sec. I of the supplementary material. In short, thin aluminum films are 
thermally evaporated onto oxidized silicon wafer substrates $\left.(120 \mathrm{~nm} \mathrm{SiO})_{2}\right)$ and subsequently potentiostatically anodized under carefully selected conditions to form an ordered system of tubular pores. To infiltrate the pores with perovskite, the precursor solution is deposited by spin coating immediately followed by annealing at $115^{\circ} \mathrm{C}$. We first address the structure of the npAl pore system by SAXS in order to quantify the average diameter of the pores $\left(2 r_{p}\right)$ and their nearest neighbor distance $\left(D_{p}\right)$. For this purpose, we measure the as prepared $\sim 1 \mu \mathrm{m}$ thick npAl films in transmission with the $\mathrm{x}$ ray beam along the surface normal [Fig. 1(a)]. The direct beam is blocked, and the intensity distribution is recorded by an area detector. The details about the setup and data analysis are presented in Sec. I of the supplementary material. The SAXS intensity is symmetric around the position of the transmitted beam [Fig. 1(b)], indicating that shape and arrangement of the pores are isotropic within the plane of the sample surface. In Fig. 1(b), the azimuthally averaged SAXS signal $I(q)$ is plotted against the scattering vector $q=4 \pi / \lambda \cdot \sin (2 \Theta / 2)$ as black circles. Here, $2 \Theta$ is the scattering angle, and $\lambda=0.62 \AA$ is the $\mathrm{x}$-ray wavelength. We interpret the SAXS intensity $I(q)$ as a product of the structure factor $S(q)$, quantifying the arrangement and in particular the interpore distance, and the squared form factor $|F(q)|^{2}$, representing the shape and size of the pores. We find that the data can be interpreted as the scattering signal of cylindrical (tubular) pores with well-defined interpore distances. An appropriate model was developed as shown below. First, we note that the form factor of isolated cylindrical pores, whose long axes are oriented with a small divergence around the direction of the incoming $\mathrm{x}$-ray beam, is of the form

$$
F^{2}(q)=\text { Constant } \cdot q^{-1} \cdot \sum_{r} p(r) \cdot\left(r \cdot \frac{J_{1}(q \cdot \sin (\alpha) \cdot r)}{q \cdot \sin (\alpha)}\right)^{2} \cdot e^{-\sigma_{P}^{2} \cdot q^{2}} .
$$

Here, the factor of $q^{-1}$ results from the finite angle dispersion of the pore axes (supplementary material, Sec. II). Polydispersity of the pore radius is accounted for by discrete sum averaging using a Gaussian pore radius distribution function $p(r) . J_{1}$ is the Bessel function of the first order and $\alpha$ is the angle between the pore axis and the scattering vector $q$. Finally, in order to account for pore wall roughness, a
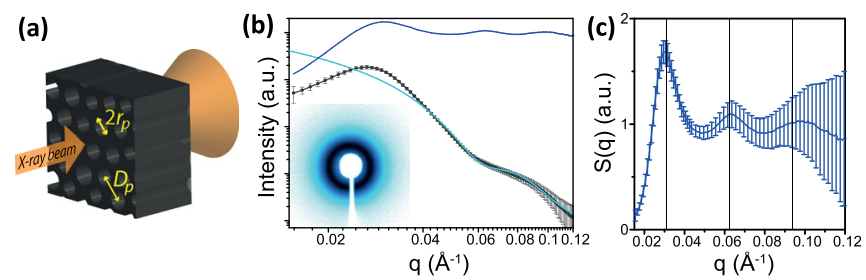

FIG. 1. SAXS analysis of nanoporous alumina films. (a) Scheme of the SAXS scattering geometry. The sample is oriented perpendicular to the incident beam. Pore diameter $\left(2 r_{p}\right)$ and pore distance $\left(D_{p}\right)$ are indicated. (b) The azimuthally averaged SAXS intensity $\boldsymbol{I}(\boldsymbol{q})$ is represented in a double logarithmic plot by black circles. The squared form factor $|\boldsymbol{F}(\boldsymbol{q})|^{2}$, as calculated for slightly polydisperse cylindrical pores (cf. main text), is shown as a light blue curve. The structure factor $\boldsymbol{S}(\boldsymbol{q})$ (dark blue curve) is obtained by the division of the measured intensity $\boldsymbol{I}(\boldsymbol{q})$ by the calculated squared form factor $|\boldsymbol{F}(\boldsymbol{q})|^{2}$. Inset: The two dimensional SAXS intensity distribution. (c) The structure factor $\boldsymbol{S}(\boldsymbol{q})$ in a linear plot. The periodicity marked by vertical lines corresponds to a characteristic interpore distance of $20 \mathrm{~nm}$. gradient of the scattering length density at the pore walls with the breadth $\sigma_{P}$ is included as an exponential damping term, as proposed by Engel et al. ${ }^{16}$

We find that an average pore radius, pore size polydispersity, and pore wall roughness of $r_{P}=5.85 \mathrm{~nm}$, $\sigma_{r}=1.15 \mathrm{~nm}$, and $\sigma_{P}=0.5 \mathrm{~nm}$, respectively, yield a satisfying agreement of $|F(q)|^{2}$ with the data, cf. light blue curve in Fig. 1(b). Thus, the first result from this analysis is that pore diameters of $11.7 \mathrm{~nm}$ can be achieved in $\mathrm{npAl}$. The form factor model, which neglects the structure factor $S(q)$, i.e., the contribution of the pore ordering, reproduces only the data at larger $q>0.04 \AA^{-1}$. In order to reproduce the full data range, we isolate the structure factor $S(q)$ from the measured intensity $I(q)$ by division, i.e., $S(q)=I(q) /|F(q)|^{2}$. The structure factor $S(q)$ obtained this way approaches an average value of 1 for larger q, as expected. Remarkably, $S(q)$ exhibits characteristic damped oscillations including the first and second order peaks, as indicated by the vertical lines in the linear representation of $S(q)$ in Fig. 1(c). The peak repeat distances and first order peak width of $\sim 0.03 \AA^{-1}$ and $\sim 0.01 \AA^{-1}$ can be read off, corresponding to a pore center to center distance and a lower limit of the correlation length among different pores of $20 \mathrm{~nm}$ and $\sim 65 \mathrm{~nm}$, respectively. Thus, our SAXS results suggest that the investigated npAl films exhibit ordered arrays of tubular pores which are oriented with their axes along the surface normal of the films. This clearly distinguishes npAl from npSi, which showed little ordering in previously published SAXS data. ${ }^{14}$

Now, we address the perovskite NP size and shape within the pores. For this purpose, we use microbeam highenergy WAXS with the porous film aligned parallel to the incoming X-ray beam. This geometry [Fig. 2(a)] is possible due to an $\sim 100 \mathrm{keV}$ x-ray beam of $\sim 1.6 \mu$ m height (FWHM) at the sample position, which is available at the high-energy beamline P07, Petra III at DESY (Deutsches ElektronenSynchrotron). ${ }^{17}$ The geometry has two compelling advantages. First, it promotes a strong scattering signal, since the beam passes the porous film throughout a sample length of $\sim 1 \mathrm{~cm}$ maximizing the number of scatterers in the beam. Consequently, the two dimensional diffraction pattern of an npAl film infused with methylammonium lead tribromide ( $\mathrm{MAPbBr}_{3}$ ), shown in Fig. 2(b), features pronounced powder rings stemming from the perovskite NP, which clearly stand out against the background of the substrate, $\mathrm{npAl}$, and air scattering. Second, the choice of geometry allows us to detect anisotropic structures.

In Fig. 2(b), the sample horizon, i.e., the part of the diffraction pattern that only contains in-plane information, is depicted as a red dashed line, denoted as $\|$, while pure outof-plane diffraction, denoted as $\perp$, is found along the blue vertical dashed line. In order to probe anisotropy, circular sectors of $\pm 15^{\circ}$ around these main axes are analyzed separately. The background corrected and azimuthally averaged data of the out-of-plane sector $S_{1}$ (blue dashed curve) and the in-plane sector $S_{2}$ (red solid curve) are shown in Fig. 2(c). The diffraction pattern can be indexed to a cubic unit cell, in accordance with bulk $\mathrm{MAPbBr}_{3}$ experiments at room temperature. ${ }^{18}$ We observe a strong anisotropy in peak width, i.e., the in-plane signal shows more pronounced peak broadening compared to the out-of-plane direction, cf. inset 
(a)

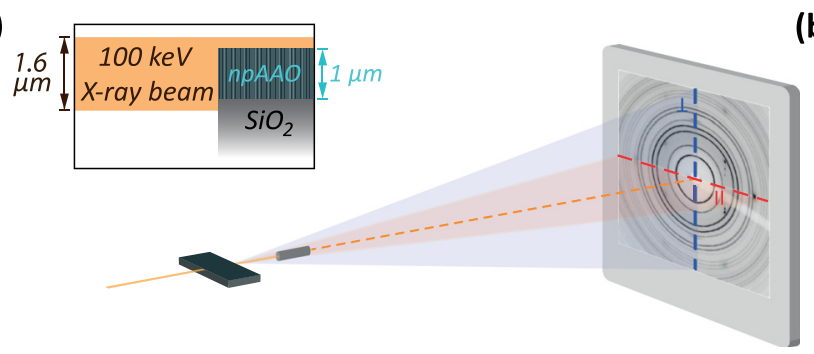

(c)

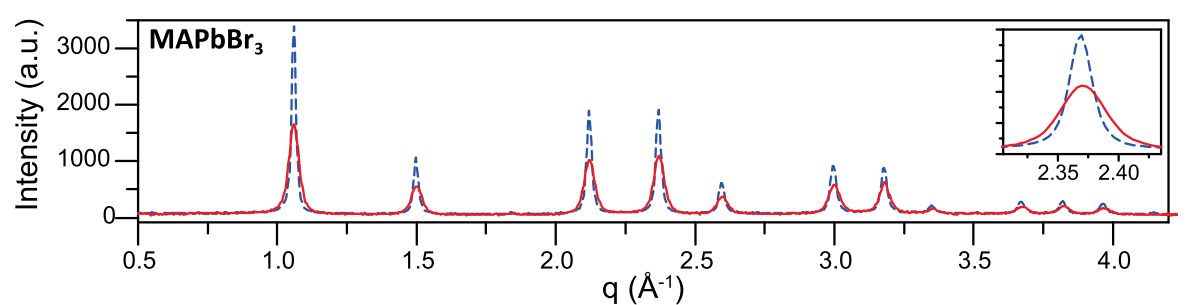

(d)

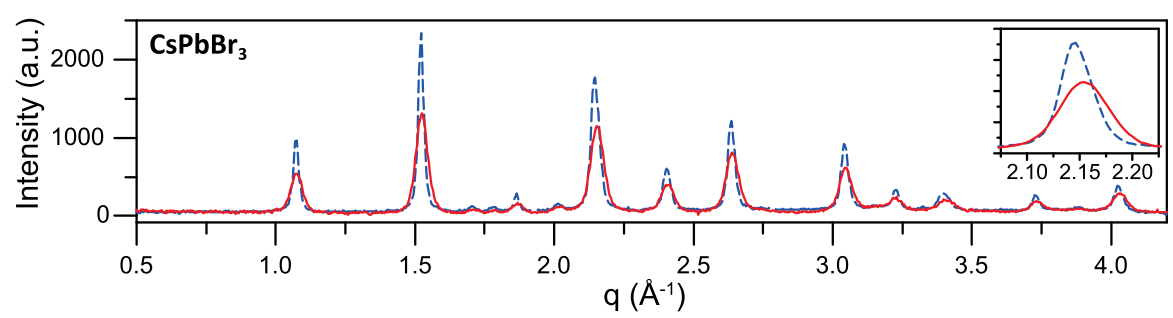

FIG. 2. Peak width analysis for crystallite size and shape. (a) Scattering geometry schematic of the high-energy microbeam WAXS experiment. The $\sim 1 \mu \mathrm{m}$ thick porous layer is aligned parallel to the incident beam with a vertical FWHM of $\sim 1.6 \mu \mathrm{m}$ (inset). (b) Scattered intensity of an $\sim 1 \mu \mathrm{m}$ thick npAl film with $\mathrm{MAPbBr}_{3} \mathrm{NP}$ (sample length $\sim 6 \mathrm{~mm}$ ). Blue and red dashed lines indicate the sections dominated by out-of-plane $(\perp)$ and in-plane $(\|)$ scattering, respectively; dotted black lines display the sectors used for further analysis. The inset shows the definition of the crystallite diameter $d_{c}$ and crystallite length $L_{c}$. (c) Background corrected intensity averaged azimuthally over the out-of-plane sector $\mathrm{S}_{1}$ (blue dashed line) and the in-plane sector $\mathrm{S}_{2}$ (red solid line). The inset highlights the increased peak broadening in the in-plane direction illustrated by the (210) peak. (d) Out-of-plane (blue dashed line) and in-plane diffraction intensity (red solid line) of $\mathrm{CsPBr}_{3}$ crystallites confined to a npAl layer. The data were processed analogously to (b) and (c). The inset shows the double peak consisting of the (040) and (202) diffraction.

of Fig. 2(c). The same signature is found while moving the film vertically with respect to the $\mathrm{x}$-ray beam-profile in steps of $0.1 \mu \mathrm{m}$ for 20 slightly different sample positions, see supplementary material, Fig. S2. This implies that in the out-ofplane direction, i.e., along the surface normal, the NP crystallite size is larger. A quantitative analysis of crystallite size and lattice spacing is performed by fitting the data by the sum of 12 Gaussian peaks. The set of fit parameters is the cubic lattice spacing $a$, and 12 values for the FWHM of the peaks. No systematic increase in the peak width with the Miller index is observed (cf. supplementary material Fig. S3), indicating that finite size effects are indeed the main contribution to the peak broadening rather than lattice stress. We calculate the anisotropic crystallite size according to the Scherrer equation ${ }^{19}$ from the peak positions and the FWHM, after correction for instrumental broadening. We find a mean out-of-plane and in-plane crystallite length of $L_{c}=27 \pm 3 \mathrm{~nm}$ and $d_{c}=13 \pm 1 \mathrm{~nm}$, revealing a ratio of long to short axis of about 2:1. These values result from averaging the crystallite sizes obtained from the nine diffraction peaks with the lowest fit uncertainty for the eight measurement positions with the strongest perovskite signal and both equivalent sectors, respectively $\left(S_{1}\right.$ and $S_{3}$ for the out-of-plane value and $S_{2}$ and $S_{4}$ for the in-plane value). We also calculate the average lattice parameter and obtain an out-of-plane and an in-plane lattice parameter of $a_{\perp}=5.931 \pm 0.001 \AA$ and $a_{\|}=5.926 \pm 0.001 \AA$, respectively, i.e., we find a slightly anisotropic lattice constant $(0.1 \%)$ that is marginally larger than the bulk constant $a=5.9195 \pm 0.0001 \AA$ reported by Elbaz et al. (by $0.2 \%$ and $0.1 \%$ for $a_{\perp}$ and $a_{\|}$, respectively). ${ }^{18}$

Similar measurements were performed with an $\sim 1 \mu \mathrm{m}$ thick npAl layer with cesium lead tribromide $\left(\mathrm{CsPbBr}_{3}\right) \mathrm{NP}$. The extracted intensity profiles are shown in Fig. 2(d), while the two dimensional raw data are shown in Fig. S4 in the supplementary material. $\mathrm{CsPbr}_{3} \mathrm{NPs}$ exhibit an orthorhombic crystal structure resembling the bulk structure reported by Stoumpos et al. for room temperature measurements. ${ }^{20} \mathrm{In}$ orthorhombic powder data, many peaks split compared to a cubic system. For simplicity, we restrict the peak width analysis to intensity maxima that consist of only two diffraction peaks, i.e., (020) and (101) and (040) and (202). The details about the fitting procedure are presented in Sec. IV of the supplementary material. We find average crystallite dimensions of $L_{c}=26 \pm 5 \mathrm{~nm}$ and $d_{c}=13 \pm 2 \mathrm{~nm}$, respectively. All values are very similar to the $\mathrm{MAPbBr}_{3}$ system, suggesting that the shape anisotropy is determined largely by the geometric pore constraints and the identical wet processing. For the out-of plane and in-plane lattice constants, we obtain $a_{\perp}=8.262 \pm 0.002 \AA$ and $a_{\|}=8.246 \pm 0.002 \AA$ and accordingly $b_{\perp}=11.761 \pm 0.003 \AA$ and $b_{\|}=11.738 \pm 0.003 \AA$ and $c_{\perp}=8.216 \pm 0.002 \AA$ and $c_{\|}=8.200 \pm 0.002 \AA$. 
The close match of the crystallite size and anisotropy between two different perovskite systems in identically processed npAl films suggests that using tubular alumina pores as a template for crystallization is an effective method to shape metal halide perovskite NP. This is further supported by the similarity of the obtained lateral sizes of crystallites and pores, indicating that the crystallite growth is directly limited by the pore walls. Distinct advantage of this method is that the larger crystallite size along the pore axis most likely favors high mobility charge carrier transport and recombination within the $\sim 40 \mathrm{~nm}$ thick active layers of our previously reported LEDs, ${ }^{14}$ whereas the reduced lateral dimension is still small enough to account for the observed blue shifted emission. The observed mean crystallite diameter of $13 \mathrm{~nm}$ led to blue shifts of the PL peak emission wavelengths of $10 \mathrm{~nm}$ and $12 \mathrm{~nm}$ for $\mathrm{MAPbBr}_{3}$ and $\mathrm{CsPbBr}_{3}$, respectively. In order to enhance the aspect ratio of the nanoparticles further and thereby promote efficient charge carrier transport, methods to increase the perovskite filled portion of the pore volume should be explored. Using higher concentrations of the precursors and optimizing the spin-coating protocol as suggested by Kwon et al., ${ }^{21}$ as well as performing the infusion process in vacuum, might lead to improved device performance. Additionally, providing an excess of precursor material and subsequently removing potential bulk accumulations from the top of the layers might allow for ideal porefilling, as observed by Ashley et al. ${ }^{22}$ for pore sizes of down to $50 \mathrm{~nm}$.

In summary, we have shown that npAl films are effective templates for nanoparticle synthesis from a metalhalide perovskite precursor solution. The procedure yields two dimensional arrays of parallel oriented perovskite nanorods with aspect ratios of 2:1. Accessing this information was possible by combining conventional SAXS with highenergy WAXS using a microbeam with a vertical FWHM of $1.6 \mu \mathrm{m}$ available at Petra III, DESY. The measurement with the films aligned parallel to the incoming microbeam allows for a straightforward extraction of out-of-plane and in-plane information from one two dimensional diffraction pattern, as it provides data for $q_{\|}=0$ as well as for $q_{\perp}=0$. This makes the method a valuable complement to the powerful but computationally more extensive grazing incidence geometry. ${ }^{23,24}$ The pores, and therefore also the nanorod arrays, are rather densely packed and well ordered. Besides the merit of this material for application in LEDs, recent demonstrations of superfluorescence from perovskite NP superlattices ${ }^{25}$ as well as reported lasing from perovskite nanowires ${ }^{26}$ encourage future investigations of optoelectronic coupling effects in perovskite nanorod arrays. Our perovskite filled $\mathrm{npAl}$ layers might constitute a versatile template for studying such effects, as interpore distance tunability and pore size tunability are well investigated for porous alumina films, ${ }^{27,28}$ and a two-step anodization process may promote even higher ordered monodisperse nanopore arrays. ${ }^{29}$

See supplementary material for details about sample preparation, the x-ray setups and data analysis and interpretation, and additional WAXS data of the perovskite filled films for different sample positions.
Parts of this research were carried out at PETRA III at DESY. We would like to thank Uta Rütt and Florian Bertram for their support at beamline P08 and Martin von Zimmermann and Ann-Christin Dippel for assistance at beamline P07. We gratefully acknowledge financial support from the Bavarian Ministry for Science through the initiative "Solar Technologies Go Hybrid" (SolTech), the Nanosystems Initiative Munich (NIM), and the Deutsche Forschungsgemeinschaft through the SFB grant 1032 project A7. This work was supported by the European Research Council Starting Grant "GEL-SYS" (Grant Agreement No. 757931) and a startup grant of the Linz Institute of Technology (Grant Agreement No. LIT013144001SEL).

${ }^{1}$ L. Protesescu, S. Yakunin, M. I. Bodnarchuk, F. Krieg, R. Caputo, C. H. Hendon, R. X. Yang, A. Walsh, and M. V. Kovalenko, Nano Lett. 15, 3692 (2015).

${ }^{2}$ L. C. Schmidt, A. Pertegás, S. González-Carrero, O. Malinkiewicz, S. Agouram, G. Mínguez Espallargas, H. J. Bolink, R. E. Galian, and J. Pérez-Prieto, J. Am. Chem. Soc. 136, 850 (2014).

${ }^{3}$ J. A. Sichert, Y. Tong, N. Mutz, M. Vollmer, S. Fischer, K. Z. Milowska, R. García Cortadella, B. Nickel, C. Cardenas-Daw, J. K. Stolarczyk, A. S. Urban, and J. Feldmann, Nano Lett. 15, 6521 (2015).

${ }^{4}$ S. Bai, Z. Yuan, and F. Gao, J. Mater. Chem. C 4, 3898 (2016).

${ }^{5}$ H. Huang, L. Polavarapu, J. A. Sichert, A. S. Susha, A. S. Urban, and A. L. Rogach, npg Asia Mater. 8, e328 (2016).

${ }^{6}$ A. Kostopoulou, E. Kymakis, and E. Stratakis, J. Mater. Chem. A 6, 9765 (2018).

${ }^{7}$ Y. Kim, E. Yassitepe, O. Voznyy, R. Comin, G. Walters, X. Gong, P. Kanjanaboos, A. F. Nogueira, and E. H. Sargent, ACS Appl. Mater. Interfaces 7, 25007 (2015).

${ }^{8}$ S. Kahmann, M. Sytnyk, N. Schrenker, G. J. Matt, E. Spiecker, W. Heiss, C. J. Brabec, and M. A. Loi, Adv. Electron. Mater. 4, 1700348 (2018).

${ }^{9}$ J. Pan, L. N. Quan, Y. Zhao, W. Peng, B. Murali, S. P. Sarmah, M. Yuan, L. Sinatra, N. M. Alyami, J. Liu, E. Yassitepe, Z. Yang, O. Voznyy, R. Comin, M. N. Hedhili, O. F. Mohammed, Z. H. Lu, D. H. Kim, E. H. Sargent, and O. M. Bakr, Adv. Mater. 28, 8718 (2016).

${ }^{10}$ D. N. Dirin, L. Protesescu, D. Trummer, I. V. Kochetygov, S. Yakunin, F. Krumeich, N. P. Stadie, and M. V. Kovalenko, Nano Lett 16, 5866 (2016).

${ }^{11}$ V. Malgras, S. Tominaka, J. W. Ryan, J. Henzie, T. Takei, K. Ohara, and Y. Yamauchi, J. Am. Chem. Soc. 138, 13874 (2016).

${ }^{12}$ V. Malgras, J. Henzie, T. Takei, and Y. Yamauchi, Angew. Chem.-Int. Ed. 57, 8881 (2018).

${ }^{13}$ M. Anaya, A. Rubino, T. Cristina Rojas, J. Francisco Galisteo-Lopez, M. Ernesto Calvo, and H. Miguez, Adv. Opt. Mater. 5, 1601087 (2017).

${ }^{14}$ S. Demchyshyn, J. M. Roemer, H. Groiß, H. Heilbrunner, C. Ulbricht, D. Apaydin, A. Böhm, U. Rütt, F. Bertram, G. Hesser, M. C. Scharber, N. S. Sariciftci, B. Nickel, S. Bauer, E. D. Głowacki, and M. Kaltenbrunner, Sci. Adv. 3, e1700738 (2017).

${ }^{15}$ E. Huang, M. F. Toney, W. Volksen, D. Mecerreyes, P. Brock, H. C. Kim, C. J. Hawker, J. L. Hedrick, V. Y. Lee, T. Magbitang, R. D. Miller, and L. B. Lurio, Appl. Phys. Lett. 81, 2232 (2002).

${ }^{16}$ M. Engel, B. Stühn, J. J. Schneider, T. Cornelius, and M. Naumann, Appl. Phys. A 97, 99 (2009).

${ }^{17}$ N. Schell, A. King, F. Beckmann, T. Fischer, M. Mueller, and A. Schreyer, Mater. Sci. Forum 772, 57 (2014).

${ }^{18}$ G. A. Elbaz, D. B. Straus, O. E. Semonin, T. D. Hull, D. W. Paley, P. Kim, J. S. Owen, C. R. Kagan, and X. Roy, Nano Lett. 17, 1727 (2017).

${ }^{19}$ P. Scherrer, Nachr. Ges. Wiss. Göttingen, Math.-Phys. Kl. 1918, 98 (1918).

${ }^{20}$ C. C. Stoumpos, C. D. Malliakas, J. A. Peters, Z. Liu, M. Sebastian, J. Im, T. C. Chasapis, A. C. Wibowo, D. Y. Chung, A. J. Freeman, B. W. Wessels, and M. G. Kanatzidis, Cryst. Growth Des. 13, 2722 (2013).

${ }^{21}$ H.-C. Kwon, A. Kim, H. Lee, D. Lee, S. Jeong, and J. Moon, Adv. Energy Mater. 6, 1601055 (2016). 
${ }^{22}$ M. J. Ashley, M. N. O'Brien, K. R. Hedderick, J. A. Mason, M. B. Ross, and C. A. Mirkin, J. Am. Chem. Soc. 138, 10096 (2016).

${ }^{23}$ C. M. Schleputz, S. O. Mariager, S. A. Pauli, R. Feidenhans'1, and P. R. Willmott, J. Appl. Cryst. 44, 73 (2011).

${ }^{24}$ E. L. Unger, A. R. Bowring, C. J. Tassone, V. L. Pool, A. Gold-Parker, R. Cheacharoen, K. H. Stone, E. T. Hoke, M. F. Toney, and M. D. McGehee, Chem. Mater. 26, 7158 (2014).
${ }^{25}$ G. Rainò, M. A. Becker, M. I. Bodnarchuk, R. F. Mahrt, M. V. Kovalenko, and T. Stöferle, Nature 563, 671 (2018).

${ }^{26}$ T. J. S. Evans, A. Schlaus, Y. Fu, X. Zhong, T. L. Atallah, M. S. Spencer, L. E. Brus, S. Jin, and X.-Y. Zhu, Adv. Opt. Mater. 6, 1700982 (2018).

${ }^{27}$ K. Nielsch, J. Choi, K. Schwirn, R. B. Wehrspohn, and U. Gösele, Nano Lett. 2, 677 (2002).

${ }^{28}$ W. Lee and S.-J. Park, Chem. Rev. 114, 7487 (2014).

${ }^{29}$ H. Masuda and K. Fukuda, Science 268, 1466 (1995). 